\title{
A Model to Describe the Distribution of Transmission Path Elevation Angles to the Iridium and Globalstar Satellite Systems
}

\author{
K. E. Crowe and R. A. Raines, Member, IEEE
}

\begin{abstract}
The quality of a satellite-based personal communications system (S-PCS) transmission path is heavily influenced by its elevation angle. The distribution of path elevation angles varies with a user's latitude but can be characterized by a single distribution with changing parameters. Through simulation, model fitting and regression analysis, a set of equations is presented which allows the distribution of elevation angles to both Globalstar $^{1}$ and Iridium ${ }^{2}$ systems to be determined for any point on the earth between $\pm 60^{\circ}$ latitude.
\end{abstract}

Index Terms - Mobile satellite communications, propagation models.

\section{INTRODUCTION}

I RIDIUM and Globalstar are satellite-based personal communication systems utilizing constellations of low earth orbiting satellites to augment terrestrial cellular coverage. The systems provide voice, fax, and low speed data services to hand held personal communications devices. Currently, 66 Iridium satellites are operational in six high inclination $\left(86.4^{\circ}\right)$ orbital planes at 780-km altitude. Globalstar, when operational, will consist of 48 satellites at $1414 \mathrm{kms}$ altitude in eight orbital planes at $52^{\circ}$ inclination. The path geometry and satellite visibility of both of these systems is strongly dependent on the user's latitude. Iridium's constellation structure provides minimum coverage and path elevation angles at the equator while the Globalstar architecture provides generally more visible satellites at higher elevation angles but provides little or no coverage above $75^{\circ}$ latitude.

The elevation angle of the transmission path is an important parameter in the assessment of link quality. Signal impairments from multipath interference, shadowing and blocking tend to be more severe at low elevation angles [1]. Atmospheric effects such as tropospheric scintillation, rain and gaseous attenuation are also higher for low elevation angle paths [2], [3]. The overall impact of these effects can be assessed if the distribution of elevation angles to all available satellite paths can be determined. With this information, a sample

Manuscript received March 3, 1999. The associate editor coordinating the review of this letter and approving it for publication was Dr. Z. Zvonar.

K. E. Crowe is with R1-3-D007, Queen Victoria Tce, Canberra, ACT 2600, Australia.

R. A. Raines is with the Air Force Institute of Technology, Wright-Patterson AFB (WPAFB), OH 45433-7765 USA.

Publisher Item Identifier S 1089-7798(99)07361-5.

${ }^{1}$ Globalstar is a trademark of Globalstar, L.P.

${ }^{2}$ Iridium is a registered trademark and service mark of Iridium LLC. set can be easily generated without setting up an orbital simulation package or deploying a test station. Additionally, the combination of elevation angle distribution and satellite altitude can be used to provide the distribution of path losses (neglecting atmospheric and ionospheric effects).

\section{MEthodOlOGY}

As a process of conducting a wider comparative analysis of the transmission path of the Iridium and Globalstar systems, the two constellations were simulated using Satellite Tool Kit [4] based on parameters in open literature [5]. An $8.2^{\circ}$ lower elevation angle limit was implemented for Iridium with a $10^{\circ}$ limit set for Globalstar. Satellites below this limit were deemed not to be visible. Simulations were conducted for orbit durations ranging from $4 \mathrm{~h}$ to 14 days with path data samples to all visible satellites taken at 10 and $30 \mathrm{~s}$ intervals. The resulting data sets were analyzed to compare statistical moments including average, median, skewness, kurtosis, percentiles and extreme values. It was concluded that an orbital simulation of $24 \mathrm{~h}$ with samples taken at 30-s intervals provided an adequate representation of the actual distribution of Iridium and Globalstar elevation angles. Path data was taken from 19 simulated sea-level ground stations located at $5^{\circ}$ latitude intervals from the equator to the North Pole. All of the stations were located at $135^{\circ}$ longitude, although the distribution of elevation angles was found to be independent of longitude for simulation runs of greater than $12 \mathrm{~h}$. Additionally, as the orbits are circular, the distribution of elevation angles is assumed to be identical for both the North and South hemispheres. The resulting data sets were read into Expert Fit [6], which was used to fit, test and rank the sample sets against 31 continuous distribution models. Each of the models which ranked in the top five was assessed according to the average and maximum error between the sample and the model approximation. The error was defined as a proportion of the total sample size, therefore an error at a point on the distribution of 0.005 indicated that the model distribution differed from the sample probability density function by $0.5 \%$ of the sample size. Based on this error analysis, a single distribution was selected to best represent the data across the range of latitudes. Each of the resulting distribution parameters (shape and location) was tabulated and a regression analysis conducted to characterize the changes in each of the parameters with latitude. SAS-JMP [7] was used to conduct the regression analysis on those model 
distributions which consistently provided the lowest deviation from the sample.

\section{RESULTS OF ANALYSIS}

\section{A. Iridium}

Analysis of the Iridium path elevation angles indicated the distribution was relatively unchanged between the equator and $60^{\circ}$ latitude. Outside these latitudes, the path data was influenced by the simultaneous visibility of satellites from several orbital planes. This had the effect of raising the median path elevation angle from $17^{\circ}$ for latitudes up to $60^{\circ}$ to $25.5^{\circ}$ at $85^{\circ}$ latitude. In order to determine whether the distributions of elevation angles between $\pm 60^{\circ}$ latitude could be described by a single distribution, the Kruskal-Wallis test [8] was used to determine the level of homogeneity of the sample distributions. The test statistic $(\mathrm{H})$ of 0.948 derived as part of the Kruskal-Wallis test was found to be less than the critical value of 9.210 for 0.01 level of significance. Accordingly, the hypothesis of homogeneity of the distributions of all elevation angles was supported for latitudes between 0 and $\pm 60^{\circ}$ and a single distribution could be used to describe its shape. Analysis indicated that the Johnson $S_{B}$ bounded continuous distribution defined by (1) [8] provided the most consistently accurate fit to the distribution of Iridium elevation angles between the equator and $\pm 60^{\circ}$ latitude.

$f(\theta)=\frac{\alpha_{2}(b-a)}{(\theta-a)(b-\theta) \sqrt{2 \pi}} \exp \left\{\frac{1}{2}\left[\alpha_{1}+\alpha_{2} \ln \left(\frac{\theta-a}{b-\theta}\right)\right]^{2}\right\}$.

The associated cumulative distribution is provided by (2) [8],

$$
F(\theta)=\Phi\left[\alpha_{1}+\alpha_{2} \ln \left(\frac{\theta-a}{b-\theta}\right)\right]
$$

where $\Phi$ indicates the normal cumulative distribution function defined for the value in the bracketed expression. The parameters for substitution into the distribution are as follows:

$$
\begin{aligned}
& a(\text { Lower Endpoint })=6.35 \\
& b(\text { Upper Endpoint })=108.33 \\
& \alpha_{1} \text { (Shape Parameter \#1) }=1.8 \\
& \alpha_{2} \text { (Shape Parameter \#2) }=0.845 .
\end{aligned}
$$

The maximum error between the sample sets and the Johnson $\mathrm{S}_{\mathrm{B}}$ approximation using the parameters above is 0.02 with an average of 0.006 . The distribution is valid only between the sample limits of $8.2^{\circ}$ and $90^{\circ}$. The exponential distribution also provides a consistent fit to the sample distribution and is presented as an alternative to the Johnson $S_{B}$ due to its ease of calculation and simplicity. The exponential distribution of elevation angles $(\theta)$ is described by:

$$
F(\theta)=\frac{1}{13.77} \times \exp \left[\frac{-(\theta-8.2)}{13.77}\right] .
$$

The distribution is valid for elevation angles between $8.2-90^{\circ}$, with a maximum error of 0.02 with a mean of
0.016. The cumulative distribution function for all elevation angles is provided by (4).

$$
F(\theta)=1-\exp \left[\frac{-(\theta-8.2)}{13.77}\right] \text {. }
$$

The shapes of the two probability density distributions differ markedly below $10^{\circ}$ elevation angle. For path elevation angles below $10^{\circ}$, the exponential distribution continues to rise while the Johnson $S_{B}$ distribution falls rapidly to zero at its lower limit of $6.35^{\circ}$. Depending on the application, the different lower boundary characteristics may ultimately determine which of the two distributions is more suitable.

\section{B. Globalstar}

The distribution of elevation angles for a Globalstar user is relatively stable (median: $21.4^{\circ}$ ) between the equator and $\pm 20^{\circ}$ latitude, rising to a peak (median: $27^{\circ}$ ) at $45^{\circ}$ latitude. Above this latitude, the median elevation angle drops off almost linearly until continuous satellite coverage is lost at approximately $75^{\circ}$ latitude. Although the Johnston $S_{B}$ distribution consistently provided the highest ranked approximation to the data sets up to $70^{\circ}$, the analysis was limited to $60^{\circ}$ latitude as the changes in the shape and location parameters above this latitude became difficult to describe without using more complex, higher order polynomials. Regression analysis indicated that a two-part piecewise fit covering the two latitude ranges $0-20^{\circ}$ and $20-60^{\circ}$ provided the lowest level of overall error. The equations which describe the Johnson $S_{B}$ distribution parameters between $0-20^{\circ}$ in terms of latitude $(\lambda)$ are as follows:

$$
\begin{aligned}
a & =8.10602+0.00637(\lambda) \\
b & =96.2 \\
\alpha_{1} & =1.40474+0.00679(\lambda)-0.00065(\lambda)^{2}+0.00001(\lambda)^{3} \\
\alpha_{2} & =0.8146-0.00073(\lambda) .
\end{aligned}
$$

Due to the relative stability of the distributions at low latitudes, the parameter values produced by these equations show little variation. Accordingly, the use of a simple mean of each parameter may be sufficient for some applications. In contrast, the distribution of Globalstar elevation angles at latitudes between $20^{\circ}-60^{\circ}$ changed markedly, requiring higher order coefficients to provide the required degree of accuracy. Location and shape parameters for the latitudes between $25^{\circ}-60^{\circ}$ are described by the following equations:

$$
\begin{aligned}
a= & 13.2351-0.30488(\lambda)+0.00379(\lambda)^{2} \\
b= & -293.97+30.307(\lambda)-0.71494(\lambda)^{2}+0.00525(\lambda)^{3} \\
\alpha_{1}= & -12.695+1.16742(\lambda)-0.02988(\lambda)^{2}+0.00024(\lambda)^{3} \\
\alpha_{2}= & -3.998574+0.38387(\lambda)-9.436 E-3(\lambda)^{2} \\
& +7.34 E-5(\lambda)^{3}
\end{aligned}
$$

The errors associated with using the equations derived from the regression analysis are detailed in Table I.

These errors represent the difference between the distributions derived from the use of the equations above and the sample data. Note that the errors are primarily restricted to 
TABLE I

Globalstar Elevation Angle Distribution ERrors

\begin{tabular}{c|c|c}
\hline \multirow{2}{*}{$\begin{array}{c}\text { Latitude } \\
\text { (degrees) }\end{array}$} & \multicolumn{2}{|c}{$\begin{array}{c}\text { Errors Associated With } \\
\text { Johnson } \mathbf{S}_{\mathrm{B}} \text { Approximation }\end{array}$} \\
\cline { 2 - 3 } & Average & Maximum \\
\hline 0 & 0.0022 & 0.0133 \\
\hline 5 & 0.0016 & 0.0118 \\
\hline 10 & 0.0016 & 0.0098 \\
\hline 15 & 0.0016 & 0.0127 \\
\hline 20 & 0.0020 & 0.0121 \\
\hline 25 & 0.0072 & 0.0317 \\
\hline 30 & 0.0103 & 0.0373 \\
\hline 35 & 0.0057 & 0.0276 \\
\hline 40 & 0.0085 & 0.0330 \\
\hline 45 & 0.0121 & 0.0332 \\
\hline 50 & 0.0140 & 0.0366 \\
\hline 55 & 0.0083 & 0.0262 \\
\hline 60 & 0.0230 & 0.0418 \\
\hline & &
\end{tabular}

the latitudes between $35^{\circ}-50^{\circ}$ with the greatest deviation at $45^{\circ}$. The reader is cautioned against approximating the latitude $(\lambda)$ coefficients in any of the equations described above. Any reduction of coefficient accuracy will cause substantial errors in the parameters, resulting in large errors in the distribution approximations.

\section{CONCLUSION}

Equations have been presented which allow the distribution of path elevation angles to the Globalstar and Iridium satellite systems to be characterized in terms of the user's latitude. In conjunction with existing methods of estimating signal propagation impairments, these equations may be used to predict the total levels of fade for S-PCS signals.

\section{REFERENCES}

[1] R. Akturan and W. J. Vogel, "Elevation angle dependence of fading for satellite PCS in urban areas," Electron. Lett. vol. 31, no. 14, pp. 1125-1127, May 1995.

[2] J. E. Allnutt, Satellite to Ground Radiowave Propagation. London, U.K.: Peter Peregrinus, 1989.

[3] J. Goldhirst and W. J. Vogel, "Propagation effects for land mobile satellite systems: Overview of experimental and modeling results," NASA Ref. Publ. 1274, Feb. 1992.

[4] "Satellite Tool Kit (STK) Version 4.0.5," Analytical Graphics, Inc., Malvern, PA 19355.

[5] H. Stelianos, "The use of commercial low earth orbit satellite systems to support DOD communications," Ph.D. dissertation, DTIC Ref. ADA326969.

[6] "Expert Fit Version 1.5," Averill M. Law and Associates, Tucson, AZ, 1998.

[7] "SAS JMP Version 3.4.3," SAS Inst., AS Cary, NC, 1997.

[8] A. M. Law and W. D. Kelton, Simulation Modeling and Analysis. 2nd ed. New York: McGraw-Hill, 1991. 